\title{
SSH adequacy to preimplantation mammalian development: Scarce specific transcripts cloning despite irregular normalisation LC Bui ${ }^{\dagger}$, RD Léandri ${ }^{\dagger}$, JP Renard and V Duranthon*
}

Address: UMR Biologie du Développement et de la Reproduction. INRA 78350 Jouy en Josas. France

Email: LC Bui - bui.linh-chi@jouy.inra.fr; RD Léandri - roger.leandri@jouy.inra.fr; JP Renard - jean-paul.renard@jouy.inra.fr; V Duranthon* - veronique.duranthon@jouy.inra.fr

* Corresponding author †Equal contributors

Published: 08 November 2005

BMC Genomics 2005, 6:155 doi:10.1/86/|47|-2/64-6-155
Received: 09 June 2005

Accepted: 08 November 2005

This article is available from: http://www.biomedcentral.com/I47I-2/64/6/155

(c) 2005 Bui et al; licensee BioMed Central Ltd.

This is an Open Access article distributed under the terms of the Creative Commons Attribution License (http://creativecommons.org/licenses/by/2.0), which permits unrestricted use, distribution, and reproduction in any medium, provided the original work is properly cited.

\begin{abstract}
Background: SSH has emerged as a widely used technology to identify genes that are differentially regulated between two biological situations. Because it includes a normalisation step, it is used for preference to clone low abundance differentially expressed transcripts. It does not require previous sequence knowledge and may start from PCR amplified cDNAs. It is thus particularly well suited to biological situations where specific genes are expressed and tiny amounts of RNA are available. This is the case during early mammalian embryo development. In this field, few differentially expressed genes have been characterized from SSH libraries, but an overall assessment of the quality of SSH libraries is still required. Because we are interested in the more systematic establishment of SSH libraries from early embryos, we have developed a simple and reliable strategy based on reporter transcript follow-up to check SSH library quality and repeatability when starting with small amounts of RNA.
\end{abstract}

Results: Four independent subtracted libraries were constructed. They aimed to analyze key events in the preimplantation development of rabbit and bovine embryos. The performance of the SSH procedure was assessed through the large-scale screening of thousands of clones from each library for exogenous reporter transcripts mimicking either tester specific or tester/driver common transcripts. Our results show that abundant transcripts escape normalisation which is only efficient for rare and moderately abundant transcripts. Sequencing 1600 clones from one of the libraries confirmed and extended our results to endogenous transcripts and demonstrated that some very abundant transcripts common to tester and driver escaped subtraction. Nonetheless, the four libraries were greatly enriched in clones encoding for very rare $(0.0005 \%$ of mRNAs) tester-specific transcripts.

Conclusion: The close agreement between our hybridization and sequencing results shows that the addition and follow-up of exogenous reporter transcripts provides an easy and reliable means to check SSH performance. Despite some cases of irregular normalisation and subtraction failure, we have shown that SSH repeatedly enriches the libraries in very rare, tester-specific transcripts, and can thus be considered as a powerful tool to investigate situations where small amounts of biological material are available, such as during early mammalian development. 


\section{Background}

Molecular analysis during the early period of embryonic development has long been prevented in mammals because of the scarcity of biological material, whatever the species considered. In recent years, however, technical improvements in the analysis of messenger RNAs from tiny amounts of cells have revealed the complexity of the genome expressed during the preimplantation period $[1,2]$. This complexity has been highlighted in recent publications which reported the isolation of new sequences in different mammalian species [3-6]. Although the transient and tissue-specific expression of such sequences during later development cannot formally be ruled out, they are more likely to be specifically expressed during the preimplantation stages, which has thus prevented their identification until now [7]. This underlines the need for dedicated transcriptomic tools to investigate these developmental stages. Such tools now exist for studies in mice [8], but still have to be developed for other species. Of the different strategies available to establish cDNA libraries, Suppression Subtractive Hybridisation (SSH) [9] is an efficient and widely-used PCR-based method to obtain subtracted libraries and isolate differentially expressed genes. The procedure involves two successive tester-driver hybridisation steps, the first of which induces a normalisation of tester-specific molecules, thus allowing the subsequent cloning of rare, tester-specific transcripts. Because SSH can be initiated using PCR-amplified cDNAs, it seems particularly well-suited to mammal preimplantation stage embryos which contain only a few tens of picograms of mRNAs. Furthermore, because SSH does not require previous knowledge of gene sequences, it may also be suitable for species where only a small number of sequences are available in databases.

Although it has already been used to get insight into early embryo transcriptome $[10,4,6,11]$, SSH performance in this area has not been widely documented. This is because authors usually establish only one dedicated library and provide information restricted to a few biologically relevant clones isolated from this library. Because we were interested in the more systematic establishment of SSH libraries dedicated to the analysis of early mammalian embryo development, we designed a procedure involving exogenous reporter transcripts that mimic either testerspecific or tester-driver common transcripts to enable the large scale assessment of the quality of such libraries. This procedure was applied to four independent libraries and provided information on their quality and the repeatability of the SSH procedure applied to early-stage embryos. These data were further validated by the sequencing and clustering of about 1600 clones isolated from one of these libraries. Our results show that when applied to preimplantation mammal embryos, the cDNAs of which had been pre-amplified using the SMART (Clontech patent) procedure, SSH-generated libraries repeatedly provided access to very scarce, tester-specific transcripts, despite irregular normalisation and some subtraction failures.

\section{Results and discussion}

Three of the libraries we established aimed to analyse embryonic genome transcriptional activation in rabbit and cattle (the so-called rab1, rab3 and bov1 libraries), while the fourth library (rab2) was designed for studies on early cell differentiation at the blastocyst stage in the rabbit.

In order to achieve a broad appraisal of the quality of the libraries, we decided to array several thousand clones from each library and analyse the abundance of tester-specific and tester/driver common transcripts in the subtracted libraries after bacteria transformation. However, neither strict tester-specific transcripts nor tester/driver common and equally expressed transcripts are identified at these stages in bovine and rabbit embryos, so we introduced exogenous $A$. thaliana transcripts into our biological material prior to pre-amplification and subtraction (see Fig. 1). We screened 768, 4608, 2683 and 4608 clones from the rab1, bov1, rab2 and rab3 libraries, respectively, for the presence of rare, tester-specific transcripts, using hybridisation with probes corresponding to the exogenous transcripts added. Whatever the lowest abundance of the reporter RNA $(0.001 \%$ or $0.0005 \%$ of messenger RNAs), we found clones which encoded for the scarce, tester-specific transcript in the libraries. These clones represented more than $0.1 \%$ of the colonies (Table 1 ). This result was in agreement with the initial findings of Diatchenko's group [9], but disagreed with the results published more recently by Ji et al. [12] that suggest that only abundant targets $(0.1 \%$ of messenger RNA) underwent efficient enrichment by SSH PCR, thus precluding the detection of transcription factors and receptors. Such divergent conclusions may have been due to differences in analytical sensitivity, because Ji et al. only considered subtracted cDNA smears after agarose gel electrophoresis [12], whereas we applied the hybridisation of specific, radiolabelled probes to thousands of bacterial colonies. We also found that exogenous reporter RNAs were differentially represented among the bacterial colonies, in line with their initial abundance in the tester material (Table 1 and Fig. 2). While clones encoding rare and moderately abundant tester-specific transcripts represented 0.2 to $0.5 \%$ of the clones in the libraries, abundant tester-specific transcripts were very frequently represented in the subtracted libraries (5 to $10 \%$ of clones). These results thus show that rare and moderately abundant transcripts are roughly normalised by the SSH procedure while abundant transcripts are not. This is inconsistent with the conclusions reached by both Diatchenko et al [9] and Ji et al [12], suggesting that all or nothing differentially expressed 


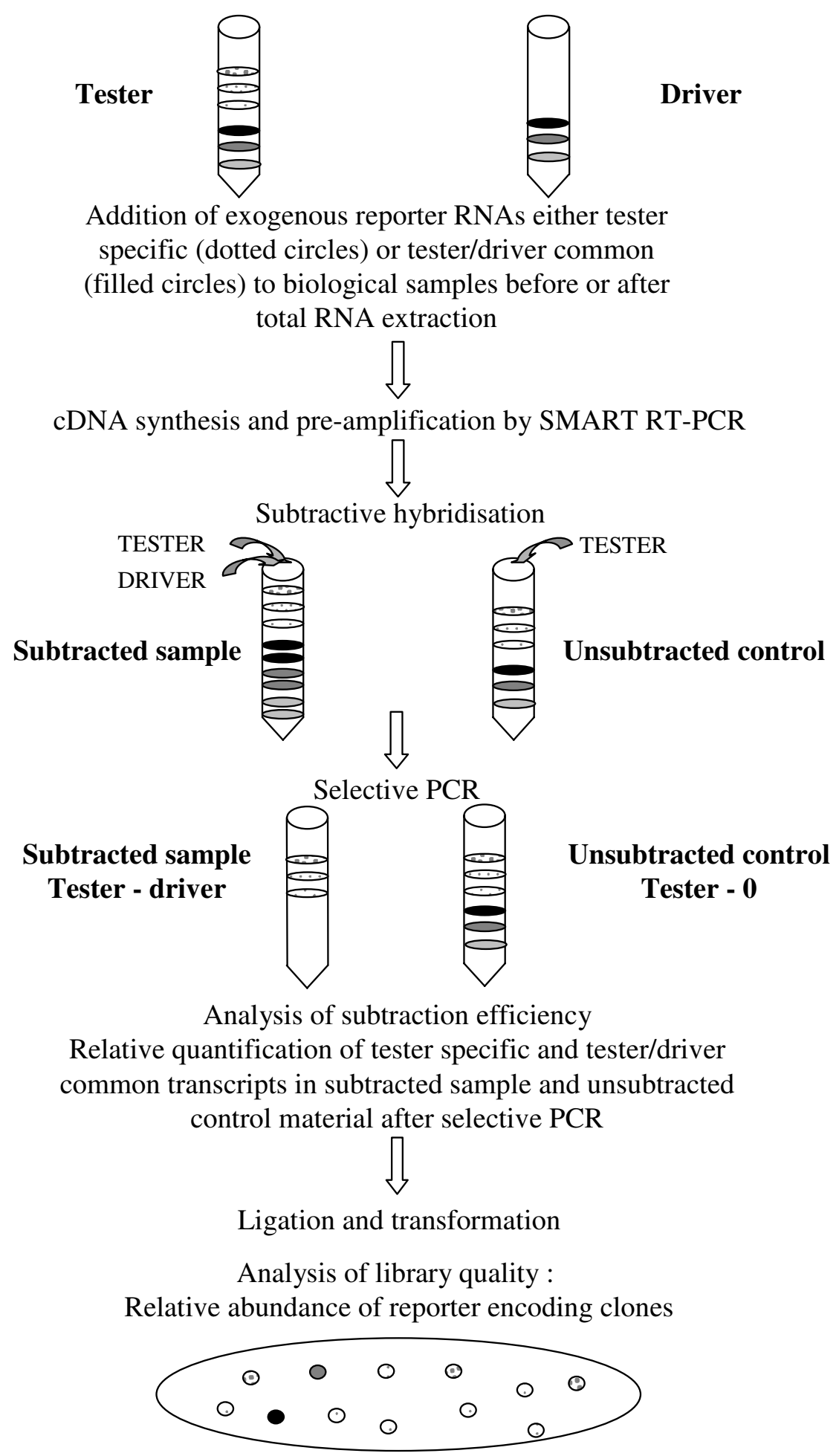

Figure I

Experimental procedure designed to check SSH efficiency. 
cDNAs were enriched to a similar final level, irrespective of their initial concentration. Here again, these divergent conclusions could be explained by differences in the experimental procedures: during our study, reporter transcripts were added to a complex biological material containing both commonly and differentially expressed transcripts, whereas tester-specific reporter DNA were added to a common cDNA used as tester and driver material by Diatchenko [9] and Ji [12]. The hybridisation kinetics during our study were probably more representative of most biological situations. Moreover, two other experimental approaches allowed us to conclude that the unequalized abundance of exogenous transcripts in our libraries was representative of the behaviour of endogenous transcripts.

First of all, we picked seven clones at random from the rab2 library that did not encode for exogenous transcripts, and analysed their abundance in the library by hybridising each radiolabelled insert to 2683 clones from the library. Three of them were present only once, the others (3L22, 3P11, 3I20 and 3C24) were found 29, 63, 152 and 362 times, respectively. The most abundant of these clones (3C24) thus represented $13.5 \%$ of the clones in the rab2 library. Its sequence corresponded to a fragment of rabbit mitochondrial 16S rRNA (nucleotide 1402 to 1899, Accession number AJ001588).

Secondly, we systematically sequenced 1920 clones from the rab2 library that did not hybridise to the 3C24 insert. From these clones, 1582 "good quality" sequences were assembled into 651 distinct contigs, of which 447 were singlet contigs. The depth of the 204 remaining contigs ranged from 2 (98 contigs) to 135 (1 contig). Only 14 contigs contained more than 10 sequences, two of them corresponding to A. Thaliana tester-specific exogenous transcripts (initial abundance 0.01 and $0.002 \%$ ). These 14 contigs totalized 638 sequences. Surprisingly, the deepest contig - that containing $135(8.5 \%)$ of the sequenced clones - encoded for the complete mitochondrial 16S rRNA, with a high prevalence of sequences bordering the $3 \mathrm{C} 24$ fragment. Finally, about $22 \%$ of the rab2 library clones encoded for this mitochondrial cDNA.

Sequence data validated the results obtained by hybridisation for tester-specific exogenous transcripts. We found $86 / 1582(5.43 \%)$ clones encoding for the abundant reporter tester-specific transcript, $14 / 1582(0.88 \%)$ clones encoding for the moderately abundant one and 6/1582 $(0.38 \%)$ for the rare one, these results being in agreement with hybridisation results (Table 1). Sequencing also confirmed the results obtained for common transcripts in the rab2 library, since no sequenced clone corresponded to these exogenous transcripts (Table 1). However, "commonly expressed transcript" elimination seemed some- what variable in the four libraries we analysed (Table 1). In the worst case (rab3 library, moderate transcript initial abundance), with respect to transcripts of the same initial abundance, we observed five-fold less representation in the library for the tester/driver commonly expressed transcript when compared with the tester-specific transcript (Table 1).

The normalisation failures observed for abundant exogenous reporter-transcripts probably reflected the results concerning endogenous transcripts: the coexistence of a majority of singlet contigs (68\%) with a few, very deep contigs.

In order to obtain more information about highly redundant endogenous sequences in the libraries, we returned to the 3L22, 3P11, 3I20 and 3C24 transcripts. Their high level of representation in the library suggested that they encode for abundant transcripts, but the mitochondrial nature of 3C24 rendered tester-specific expression highly unlikely. We thus analysed the relative abundance of these four cDNAs in tester and driver unsubtracted materials. Semi-quantitative analysis (see methods) revealed that these four clones encode for very abundant CDNA in the tester material : they represented about $0.11,0.6,2.45$ and $3.7 \%$ (for 3P11,3I20, 3L22 and 3C24 respectively) of blastocyst cDNAs. They thus constituted a fourth category of transcripts that we did not mimic with the exogenous A. thaliana transcripts. Two of them (3P11 and 3I20) were tester-specific, but the others (3L22 and 3C24) were expressed in both tester and driver, with double their relative abundance in tester than in driver (3L22 and 3C24 representing respectively about 1.2 and $1.5 \%$ of morula driver CDNAs). It thus appears that very abundant testerspecific transcripts escape normalisation, whereas very abundant transcripts expressed in both tester and driver escape both subtraction and normalisation.

Under these conditions, the few deep contigs we obtained by sequencing the library could correspond to either abundant or very abundant tester-specific transcripts escaping normalisation, or to very abundant commonly expressed transcripts escaping both subtraction and normalisation. These normalisation (and possibly subtraction) failures resulted in a significant proportion of highly redundant sequences $(638 / 1582$, or $40 \%)$ in the library.

In view of the considerable concordance between sequencing and hybridisation data, we assumed that the addition of exogenous reporter transcripts and analysis of their representation in the library would ensure reliable monitoring of SSH performance. However, the very high representation of mitochondrial 16S rRNA in the library showed that the frequency we chose to mimic abundant transcripts underestimated the representation of certain 
Table I: Proportion of reporter transcripts encoding clones in the subtracted libraries

\begin{tabular}{|c|c|c|c|c|}
\hline & Library & $\begin{array}{c}\text { Initial abundance (\%) } \\
\text { Tester specific (bold) } \\
\text { Tester/Driver Common (standard) }\end{array}$ & $\begin{array}{l}\text { Hybridised/analysed } \\
\text { clones }\end{array}$ & $\begin{array}{c}\text { Proportion in } \\
\text { subtracted library (\%) }\end{array}$ \\
\hline \multirow{4}{*}{ Rare transcripts } & $\mathrm{rabl}$ & \# & $\#$ & \# \\
\hline & bovl & 0,001 & $13 / 4608$ & 0,28 \\
\hline & $\mathrm{rab2}$ & 0,0005 & $3 / 2683$ & 0,11 \\
\hline & rab3 & 0,0005 & $14 / 4608$ & 0,3 \\
\hline \multirow[t]{8}{*}{ Moderate transcripts } & $\mathrm{rabl}$ & 0,005 & $5 / 768$ & 0,65 \\
\hline & & 0,005 & $0 / 768$ & - \\
\hline & bovl & 0,005 & $24 / 4608$ & 0,52 \\
\hline & & 0,005 & $3 / 4608$ & 0,06 \\
\hline & rab2 & 0,002 & $22 / 2683$ & 0,82 \\
\hline & & 0,002 & $0 / 2683$ & - \\
\hline & rab3 & 0,002 & I I/4608 & 0,24 \\
\hline & & 0,002 & $2 / 4608$ & 0,043 \\
\hline \multirow[t]{8}{*}{ Abundant transcripts } & $\mathrm{rabl}$ & 0,05 & $82 / 768$ & 10,7 \\
\hline & & 0,05 & $10 / 768$ & 1,3 \\
\hline & bovl & 0,05 & $195 / 4608$ & 4,2 \\
\hline & & 0,05 & $4 / 4608$ & 0,086 \\
\hline & $\mathrm{rab} 2$ & 0,01 & $147 / 2683$ & 5,48 \\
\hline & & 0,01 & $0 / 2683$ & - \\
\hline & rab3 & 0,01 & $256 / 4608$ & 5,55 \\
\hline & & 0,01 & $25 / 4608$ & 0,54 \\
\hline
\end{tabular}

\#: not introduced in library

-: no clone detected among the screened clones

transcripts in our biological material. 16 SrRNA was found to represent a high proportion (1.5 to $3.7 \%$ ) of unsubtracted cDNAs, which could be correlated to the very rapid growth [13] and marked metabolic activity of the blastocyst in the rabbit species, which certainly requires strong mitochondrial activity during the early stages of development. Mitochondrial transcripts have been shown to represent as much as $23 \%$ of polyadenylated RNAs in mouse blastocysts [14]. Such quantitative data are not available in the rabbit.

Our results show that application of the SSH procedure to early development permits the isolation of scarce testerspecific transcripts despite irregular normalisation and some subtraction failures concerning very abundant transcripts.

Taken together, these data confirmed and extended previous reports showing that whatever the origin of the clones picked from SSH constructed libraries - random choice or selection by differential screening - some of them are heavily represented among the sequenced clones $[[9,10,4,15]$, and $[16]]$. Since this unequalized representation has been reported in both SMART-preamplifiedcDNA subtracted libraries $[10,4,16]$ and non preamplified cDNA libraries [15], it should not be considered as a con- sequence of the CDNA preamplification step but rather as an intrinsic defect in the SSH procedure.

\section{Conclusion}

This study showed that SSH libraries dedicated to early mammalian development are greatly enriched in testerspecific transcripts. They are only partially normalized, with transcript equalization being restricted to rare and moderately abundant transcripts. Very abundant transcripts common to both tester and driver may escape both normalisation and subtraction, giving rise to abundant background clones in these libraries. The differential expression of genes represented by redundant clones in subtracted libraries should therefore be checked very carefully.

These conditions were however compatible with the isolation of very rare tester-specific transcripts $(0.0005 \%$ of messenger RNA) in the libraries. Under these conditions, SSH produced reproducible results in terms of rare stagespecific transcript isolation and can thus be considered as a tool of considerable potential when studying the onset of mammalian development. 


\section{Methods \\ Developmental stages used for the construction of the libraries}

The following stages were used in tester-driver subtractions:

- for rabbit embryonic activation: early morulae (16-32 cell stage) - one cell stage (rab1 library) and early morulae - 4 cell stage (rab3 library).

- for cattle embryonic activation: early morulae - four-cell stage (bov1 library)

- for early cell differentiation in rabbit: blastocyst - late morulae (32-64 cell) (rab2 library).

\section{Embryo recovery}

Cattle embryos were obtained by in vitro oocyte maturation, fertilization and embryo culture as described by Pavlok et al. [17] and Parrish et al. [18]. Four-cell and morulae stage embryos were recovered at 41 and 120 hours post-insemination respectively, from early two-cellcleaved embryos picked up at 32 hours post-insemination.

For rabbit embryos, all tester materials contained embryos produced both in vivo and in vitro, whereas driver materials contained only embryos produced in vivo. In vivo onecell, four-cell, early morulae, late morulae and blastocyst stage embryos were recovered at 19, 32, 50, 65 and $90 \mathrm{~h}$ post-coitum $(\mathrm{h} p c)$, respectively. In vitro embryos were recovered at the one cell stage ( $19 \mathrm{~h}$ post-coitum) from superovulated females treated as described by Henrion et al. [19]. They were cultured from the one cell stage onwards (19 hpc) until the early morula (58 hpc) and blastocyst (100 $\mathrm{h} p c)$ stages respectively in four different culture media: B2 medium (Laboratoire C.C.D.), B2 medium plus $2.5 \%$ foetal calf serum, ISM1-ISM2 sequential media (Medicult.) and G1-G2 sequential medium (JCD sa). In the latter two cases, the sequence used for embryo culture mimicked that used in human IVF in terms of the timing of genome transcriptional activation: embryos were cultured in the first medium until the 8 cell stage in ISM1 and until the early morula stage in G1, i.e. just before and just after the onset of embryonic genome transcription respectively, then transferred to the second medium until the stage of interest.

\section{RNA extraction}

Total RNA was extracted from batches of embryos ( $\mathrm{n}=$ 200 to 450 embryos) using the RNeasy Mini Kit (Qiagen, CA, USA) with a DNAse I treatment $\left(37^{\circ} \mathrm{C}, 30 \mathrm{~min}\right)$. Arabidopsis Thaliana RNAs (Stratagene, Spike RNA 201, 204, 205, 208, and 209) were added as reporter exogenous RNAs at different concentrations, either specifically in tester material or in both tester and driver materials. These exogenous RNAs were added either before (rab1 and bov1 libraries) or after (rab2 and rab3 libraries) total RNA extraction.

\section{cDNAs amplification, SSH and PCR amplification of subtracted products}

The tester to driver hybridisation steps in the SSH procedure require one hundred nanograms of tester and driver cDNA, whereas a preimplantation embryo only contains a few picograms of mRNAs. For this reason, we adopted the SMART PCR CDNA amplification procedure (SMARTPCR cDNA Synthesis Kit: Clontech, Palo Alto, CA, USA) starting from embryonic total RNA. The optimum numbers of PCR cycles, checked as suggested in the SMARTPCR kit protocol, were 22 (rab1), 20 (bov1 and rab2), 23 (rab3), respectively.

SSH was performed with the PCR Select cDNA Subtraction Kit (Clontech, Palo Alto, CA, USA). The first hybridisation was performed with $15 \mathrm{ng}$ amplified tester cDNA and $450 \mathrm{ng}$ amplified driver cDNA for 10 hours at $68^{\circ} \mathrm{C}$. Following the first hybridisation, $100 \mathrm{ng}$ of fresh denatured driver cDNA was added to the sample and a second hybridisation was performed at $68^{\circ} \mathrm{C}$ overnight. The final hybridisation sample was diluted in $200 \mu \mathrm{l} 20 \mathrm{mM}$ Hepes pH 8.3, $50 \mathrm{mM} \mathrm{NaCl}, 0.2 \mathrm{mM}$ EDTA buffer. Selective PCR was performed according to the recommendations of the kit manufacturer, except that the cycle numbers in the first and nested PCR were modified as mentioned in the text.

\section{Checking "selective PCR"}

In order to check subtraction efficiency after selective PCR, relative amounts of tester-specific and tester/driver common exogenous A. Thaliana transcripts were estimated in the subtracted and control unsubtracted cDNA populations. As suggested by the kit manufacturer, this was achieved by semi-quantitative PCR amplification (gradually increasing the numbers of PCR cycles, and an analysis of amplicon intensity after agarose gel electrophoresis and ethidium bromide staining). We therefore used primers specific to A. Thaliana transcripts:

\section{5'-201 TGGGTTAAGGCTCAGGAATG \\ 3'-201 GCCAAGTGAGTTGCCAAGTT}

5'-204 AACACAATGGCTTTCGCTTT

3'-204 CAAAGCCATCAAGACAAACAAA

5'-205 TTATTAGCCGTGTGCCTGGT

3'-205 CTAGCAAACCAATGCCCTCA 


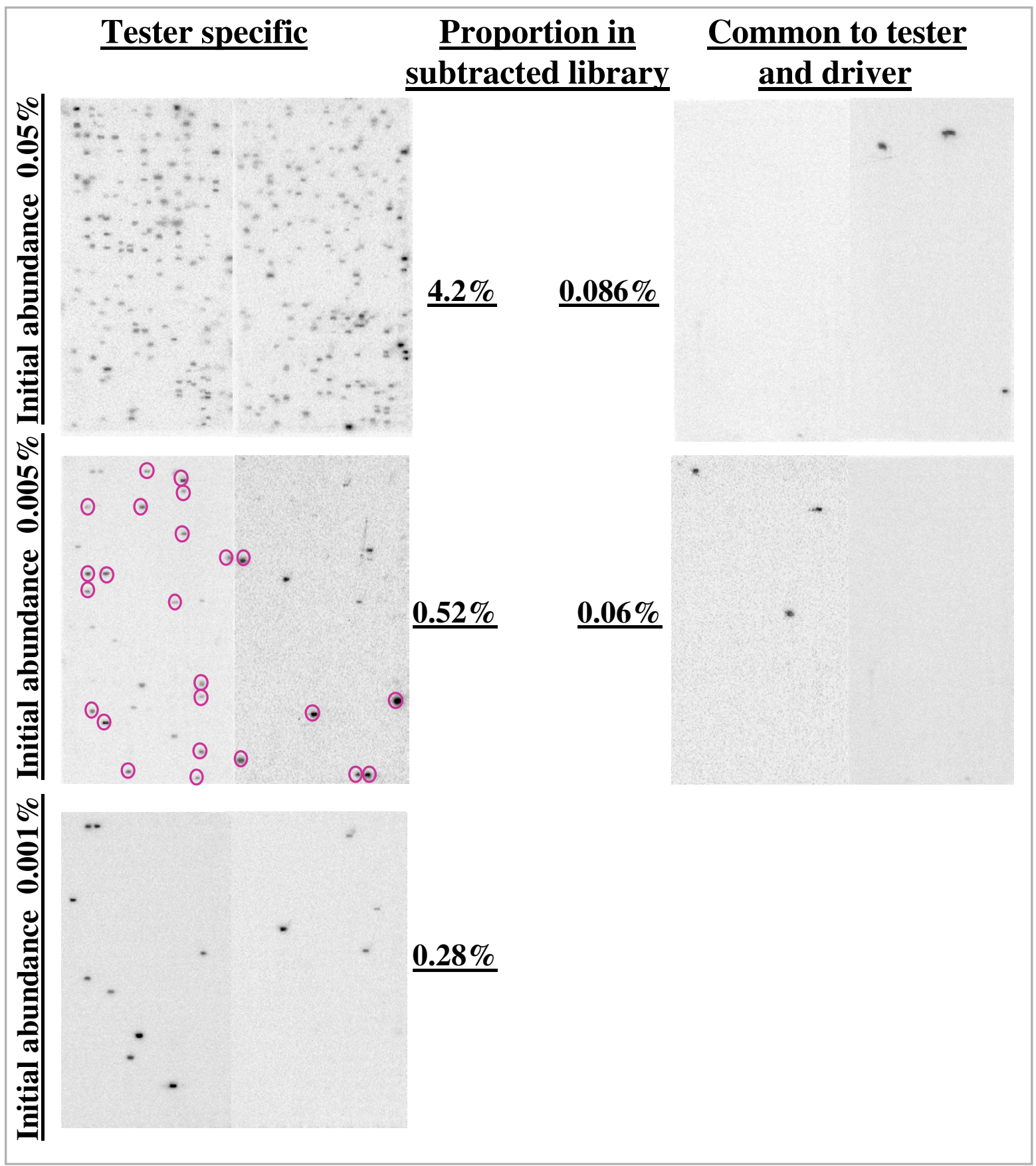

Figure 2

Abundance of reporter-transcript encoding clones in the bov I subtracted library. Screening of bovl bacteria macroarray with reporter RNA probes. Because of independent repeated hybridisations (of some membranes), only encircled clones on the left hand side (middle row) membrane should be considered as hybridising with the relevant probe. 


\section{5'-209 TTCTGTCAATGGAGGCAACA}

\section{3'-209 TGTCAAACCAGAGCTCACGA}

\section{Establishment and analysis of subtracted libraries}

PCR-amplified subtracted products (about $17 \mathrm{ng}$ ) were cloned into the pGEM-T-Easy vector (Promega) and 1/10 of the ligation volume was used to transform competent DH5 $\alpha$ Max-Efficiency E.coli bacteria (Invitrogen). After overnight culture at $37^{\circ} \mathrm{C}$, the colonies were picked and arrayed in 384 well plates. Replicates of these arrayed libraries were spotted onto nylon membranes (Hybond $\mathrm{N}+$ Amersham) placed on agar plates, and grown up for 12 hours at $37^{\circ} \mathrm{C}$. After bacteria denaturation and DNA fixation treatments, these "macroarrays" were hybridised with $32 \mathrm{P}$ radiolabelled probes corresponding to either exogenous RNAs or endogenous transcripts. Hybridisation was carried out overnight at $65^{\circ} \mathrm{C}$ in Church buffer [20].

Sequence analysis and clustering were performed as described in [21].

\section{Semi-quantitative analysis of endogenous transcript abundance in tester and driver materials}

Varying amounts (100, $300500 \mathrm{ng})$ of unsubtracted SMART-amplified tester (blastocyst) and driver (morula) cDNAs used to establish the SSH library were slot blotted on Brightstar TM-Plus membranes (Ambion). On the same membranes, various quantities (ranging from 0.01 ng to $6.25 \mathrm{ng}$ ) of cDNA inserts (3P11, 3C24, 3L22 and 3I20, respectively) were slotted. DNA inserts (3P11, 3C24, $3 \mathrm{~L} 22$ and 3I20) were labelled by random priming (Rediprime TM II Amersham). Hybridization was performed in UltrahybTM buffer (Ambion), at $42^{\circ} \mathrm{C}$, for 22 hours. Washings were performed twice in $2 \times$ SSC, $0.1 \%$ $\mathrm{SDS}$ at $42^{\circ} \mathrm{C}$ for 10 minutes, then twice in $0.1 \times \mathrm{SSC}, 0.1 \%$ SDS, $42^{\circ} \mathrm{C}$, for 30 minutes. Membranes were exposed to a phosphoscreen (Phosphorimager Amersham) for 6 hours, and hybridization signals were quantified using Imagequant (Amersham).

\section{Authors' contributions}

Véronique Duranthon is the supervisor of Linh Chi Bui and Roger Dominique Léandri, two doctoral students in the laboratory who participated directly in the experimental work. Jean Paul Renard is Director of the Laboratory.

\section{Acknowledgements}

We would like to thank Annie Chastellier and François Piumi (CRB, Jouy en Josas, France) for library arraying and bacteria membrane preparations, Cédric Cabau (Agenae) for contig assembly and Catherine Faure for her technical assistance. We also are indebted to members of the UCEA responsible for our rabbit colonies and Yvette Lavergne (BDR, INRA) for bovine embryo production.

\section{References}

I. Wang QT, Piotrowska K, Ciemerych MA, Milenkovic L, Scott MP, Davis RW, Zernicka-Goetz M: A genome-wide study of gene activity reveals developmental signaling pathways in the preimplantation mouse embryo. Dev Cell 2004, 6:133-44.

2. Hamatani T, Carter MG, Sharov AA, Ko MS: Dynamics of global gene expression changes during mouse preimplantation development. Dev Cell 2004, 6: | 77-3|.

3. Goto T, Jones GM, Lolatgis N, Pera MF, Trounson AO, Monk M: Identification and characterisation of known and novel transcripts expressed during the final stages of human oocyte maturation. Mol Reprod Dev 2002, 62:13-28.

4. Mohan M, Ryder S, Claypool PL, Geisert RD, Malayer JR: Analysis of gene expression in the bovine blastocyst produced in vitro using suppression-subtractive hybridization. Biol Reprod 2002, 67:447-53.

5. Pacheco-Trigon S, Hennequet-Antier C, Oudin JF, Piumi F, Renard JP, Duranthon V: Molecular characterization of genomic activities at the onset of zygotic transcription in mammals. Biol Reprod 2002, 67: 1907-18.

6. Yao YQ, Xu JS, Lee WM, Yeung WS, Lee KF: Identification of mRNAs that are up-regulated after fertilization in the murine zygote by suppression subtractive hybridization. Biochem Biophys Res Commun 2003, 304:60-6.

7. Ko MS, Kitchen JR, Wang X, Threat TA, Hasegawa A, Sun T, Grahovac MJ, Kargul GJ, Lim MK, Cui Y, Sano Y, Tanaka T, Liang Y, Mason S, Paonessa PD, Sauls AD, DePalma GE, Sharara R, Rowe LB, Epigg J, Morrell C, Doi H: Large-scale cDNA analysis reveals phased gene expression patterns during preimplantation mouse development. Development 2000, 127:1737-49.

8. Carter MG, Hamatani T, Sharov AA, Carmack CE, Qian Y, Aiba K, Ko NT, Dudekula DB, Brzoska PM, Hwang SS, Ko MS: In situ-synthesized novel microarray optimized for mouse stem cell and early developmental expression profiling. Genome Res 2003, 13:|0||-2|.

9. Diatchenko L, Lau YF, Campbell AP, Chenchik A, Moqadam F, Huang B, Lukyanov S, Lukyanov K, Gurskaya N, Sverdlov ED, Siebert PD: Suppression subtractive hybridization: a method for generating differentially regulated or tissue-specific cDNA probes and libraries. Proc Natl Acad Sci USA 1996, 93:6025-30.

10. Robert C, Gagne D, Bousquet D, Barnes FL, Sirard MA: Differential display and suppressive subtractive hybridization used to identify granulosa cell messenger rna associated with bovine oocyte developmental competence. Biol Reprod 200I, 64: $1812-20$.

II. Fayad T, Levesque V, Sirois J, Silversides DW, Lussier JG: Gene expression profiling of differentially expressed genes in granulosa cells of bovine dominant follicles using suppression subtractive hybridization. Biol Reprod 2004, 70:523-33.

12. Ji W, Wright MB, Cai L, Flament A, Lindpaintner K: Efficacy of SSH $P C R$ in isolating differentially expressed genes. BMC Genomics 2002, 3: 12.

13. Daniel JC: Early growth of rabbit trophoblast. Am Natural 1987, 48(899):85-98.

14. Piko L, Taylor KD: Amounts of mitochondrial DNA and abundance of some mitochondrial gene transcripts in early mouse embryos. Dev Biol 1987, I 23:364-74.

15. Bauersachs S, Rehfeld S, Ulbrich SE, Mallok S, Prelle K, Wenigerkind $H$, Einspanier R, Blum $H$, Wolf $E$ : Monitoring gene expression changes in bovine oviduct epithelial cells during the oestrous cycle. J Mol Endocrinol 2004, 32:449-66.

16. Boyer A, Lussier JG, Sinclair AH, McClive PJ, Silversides DW: Presertoli specific gene expression profiling reveals differential expression of PptI and Brd3 genes within the mouse genital ridge at the time of sex determination. Biol Reprod 2004, 71:820-7.

17. Pavlok A, Motlik J, Kanka J, Fulka J: In vitro techniques of bovine oocyte maturation, fertilization and embryo culture resulting in the birth of a calf. Reprod Nutr Dev 1989, 29:6 I I-6.

18. Parrish JJ, Susko-Parrish JL, Critser ES, Eyestone WH, First NL: Bovine in vitro fertilization with frozen-thawed semen. Theriogenology 1986, 25:59|-600.

19. Henrion G, A B, P RJ, V D: Identification of maternal transcripts that progressively disappear during the cleavage period of rabbit embryos. Mol Reprod 1997, Dev, 47(4):353-362. 
20. GM Church, W Gilbert: Genomic sequencing. Proc Natl Acad Sci USA I984, 81 : I99|-5.

21. [http://sigenae.jouy.inra.fr].

Publish with Bio Med Central and every scientist can read your work free of charge

"BioMed Central will be the most significant development for disseminating the results of biomedical research in our lifetime. " Sir Paul Nurse, Cancer Research UK

Your research papers will be:

- available free of charge to the entire biomedical community

- peer reviewed and published immediately upon acceptance

- cited in PubMed and archived on PubMed Central

- yours - you keep the copyright

Submit your manuscript here:

http://www.biomedcentral.com/info/publishing_adv.asp 